\title{
Are biomarkers indispensable in the adnexal
} \section{masses?}

\section{Editorial}

The adnexal masses (AM) constitute one of the most frequent diagnoses in current gynecology. The improvement of complementary imaging tests such as ultrasound, magnetic resonance or CT contributes to the increase of its incidence. It is estimated that $10 \%$ of patients are at risk of suffering surgery during their lifetime due to an adnexal mass. ${ }^{1}$

Although AM may be accompanied by some type of associated symptom such as pelvic pain, fever, or vomiting more often these are incidental findings The diagnosis of the AM is a real challenge for the gynecologist. Establishing the risk of malignancy of an AM will determine the follow-up and the treatment of the patient.

To establish the differential diagnosis, we will perform a detailed anamnesis, followed by a focused physical examination as well as complementary tests such as ultrasound, resonance or different laboratory tests. Frequently, the different tests complement each other to form indices such as the IOTA, ROMA or OVA1 index..$^{2-4}$

Among the biomarkers, we highlight the Ca 125 or the HE4, useful for ovarian epithelial tumors. Although the $\mathrm{Ca} 125$ is the most used individually or combined, ${ }^{5}$ HE4 could increase specificity alone or combined with $\mathrm{Ca} 125 .{ }^{6}$ On the other hand, there are other biomarkers characteristic of other histological types, including among others $\mathrm{Ca}$ 19.9, BHCG or AFP.

Biomarkers are useful for two main reasons. First, they are a fundamental tool in the differential diagnosis and diagnostic evaluation of the adnexal masses. This helps the gynecologist in making decisions. On the other hand, it is also useful in monitoring the treatment of patients. ${ }^{7}$

However, despite the proven usefulness of biomarkers, from this manuscript we want to highlight some of the limitations of any type of complementary test, and biomakers in particular.

First of all, as a complementary test, none of these markers has sufficient specificity to confirm or rule out the malignancy of an ovarian mass. For this reason, from our point of view, its value must be integrated within the context of a specific patient with a specific personal and family history. In addition, any of these values should be considered according to the clinical examination and especially, according to the echographic findings. We think that biomarkers should be performed only in those patients with ultrasound findings that suggest malignancy and no in any adnexal mass.

In the same way, biomarkers can vary for different reasons. For example, its value can be relative depending on menopausal or premenopausal patients. In this sense, sensitivity and the specificity of Ca 125, is especially low in premenopausal patients. ${ }^{89}$ Subjective evaluation and simple rules could be more sensitive even excluding Ca 125 1. In addition, it must also be considered that the value of the markers can vary according to the laboratories, with the moment
Volume 9 Issue 5 - 2018

\author{
Jorge Duro Gómez \\ Hospital Universitario Reina Sofía Córdoba, Spain
}

Correspondence: Jorge Duro Gómez, Hospital Universitario Reina Sofía Córdoba, Calle Arabista Joaquina Eguaras N ${ }^{\circ} 2$ Esc 4Iํ, CP: I40II Córdoba, Spain, Tel: 6858I0803, Email jorgedurogomez@gmail.com

Received:September 16, 2018 | Published: September 25, 2018

of the menstrual cycle, or even in benign pathologies such as endometriosis. $^{10}$

Another aspect to consider is the anxiety that could cause to request tumor markers to patients with adnexal masses with absence of any clinical or ultrasound signs of malignancy. This not only supposes an increase of the anxiety of the patient but also an increase of the associated health costs. The costs associated with the diagnosis are lower when the evaluation is carried out by an expert examiner than when the risk of malignancy index is used. ${ }^{11}$

\section{Conclusion}

According to the above we think that gynecologists have a real challenge; firstly, the results of any marker should be relativized and contextualized. On the other hand, we must restrict the request of biomarkers to those cases in which they are really suspected of malignancy or in those whose value is going to imply a therapeutic change. With this porpuse, it is essential to unify criteria and protocols among the gynecologists.

\section{Acknowledgements}

None.

\section{Conflicts of interest}

The author declares that they have no conflict of interest.

\section{References}

1. Piovano E, Cavallero C, Fuso L, et al. Diagnostic accuracy and cost-effectiveness of different strategies to triage women with adnexalmasses: a prospective study. Ultrasound Obstet Gynecol. 2017;50(3):395-403.

2. Fung ET. A recipe for proteomics diagnostic test development: the OVA1 test, from biomarker discovery to FDA clearance. Clin Chem. 2010;56(2):327-329. 
3. Wei SU, Li H, Zhang B. The diagnostic value of serum HE4 and CA125 and ROMA index in ovarian cancer. Biomed Rep. 2016;5(1):41-44.

4. Van Calster B, Van Hoorde K, Valentin L, et al. Evaluating the risk of ovarian cancer before surgery using the ADNEX model to differentiate between benign, borderline, early and advanced stage invasive, and secondary metastatic tumours: prospective multicentre diagnostic study. BMJ. 2014;349:g5920.

5. http://www.accessdata.fda.gov/cdrh_docs/reviews/K042731.pdf

6. Ghasemi N, Ghobadzadeh S, Zahraei M, et al. HE4 combined with CA125: favorable screening tool for ovarian cancer. Med Oncol. 2014;31(1):808.

7. Mongia SK, Rawlins ML, Owen WE, et al. Performance characteristics of seven automated CA 125 assays. Am J Clin Pathol. 2006;125(6):921-927.
8. Dodge JE, Covens AL, Lacchetti C, et al. Preoperative identification of a suspicious adnexal mass: a systematic review and meta-analysis. $G y$ necol Oncol. 2012;126(1):157-166.

9. Myers ER, Bastian LA, Havrilesky LJ, et al. Management of Adnexal Mass. Evid Rep Technol Assess (Full Rep). 2006;(130);1-145.

10. Grover S, Koh H, Weideman P, et al. The effect of the menstrual cycle on serum CA 125 levels: a population study. Am J Obstet Gynecol. 1992;167(5):1379-1381.

11. Meys EMJ, Jeelof LS, Ramaekers BLT, et al. Economic evaluation of an expert examiner and different ultrasound models in the diagnosis of ovarian cáncer. Eur J Cancer. 2018;100:55-64. 